\section{Highlighting preventive dentistry from an early age}

Simplyhealth Professionals is offering free access to YDEPPA (Young Denplan PreViser Patient Assessment) as an exclusive benefit to all of its member practices as part of their aim to highlight the importance of preventive dentistry from an early age. Building on the strengths of the well-established DEPPA for adults tool, YDEPPA is an online facility which provides a framework for a holistic oral health assessment of a child.

The primary benefit of YDEPPA is to support communication with young patients about their oral health and help motivate them to make improvements. YDEPPA reports offer personalised biofeedback in a patient friendly manner. A RAG (red/ amber/green) system of happy or unhappy faces is used to flag the standard of health for each component. YDEPPA focuses on three key areas for oral health: hard tissues, periodontal health and the developing dentition/occlusion. A personalised prevention plan for each patient is also produced, providing clarity for the patient and their parent or carer.

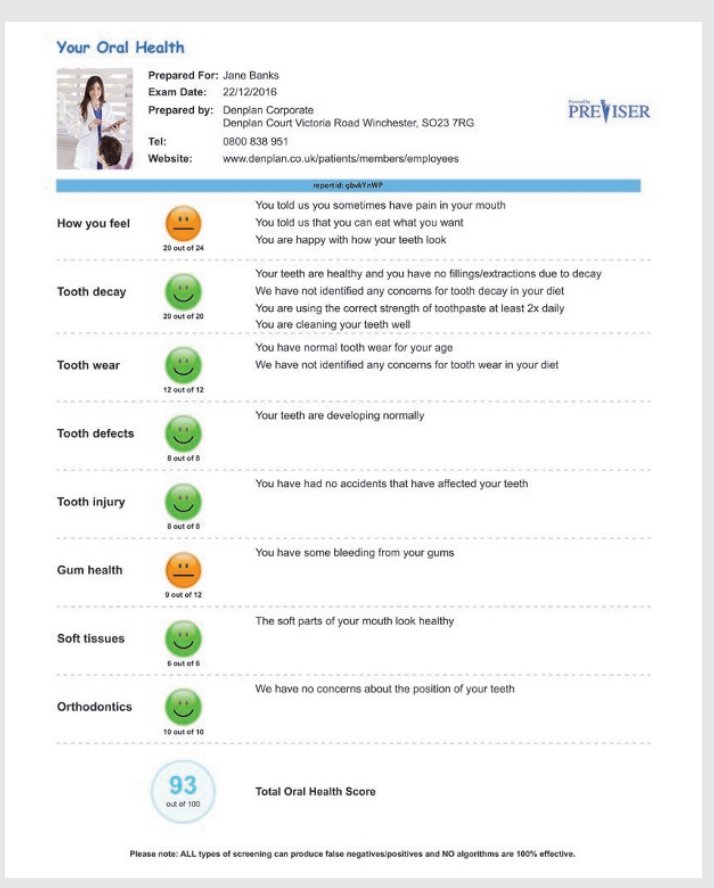

YDEPPA is very quick to complete, comprising just 14 questions. Reports can be either printed in hard copy and given to patients, or emailed to them with consent. YDEPPA reports also help patients to understand how their oral health has changed over time. Being able to view progress or changes over a longer period facilitates reinforcement of appropriate oral health related behaviour and allows clinicians the opportunity to highlight and discuss any new areas of concerns.

Free access to YDEPPA is available to Simplyhealth Professionals members. DEPPA is available free of charge to Denplan Excel members. For non-members interested in signing up to YDEPPA, a one month free trial is available for DEPPA which includes YDEPPA and access can subsequently be provided for a monthly fee based on the number of users in the practice. Practices should call 08001699962 for further information.

\section{Save time without the compromise}

In order to meet the demands of dentistry today, it's essential that you and your team can deliver restorations in a quick and efficient manner, without compromising on quality.

With Lava Esthetic Fluorescent full-contour zirconia from 3M Oral Care, you can do just this.

The material offers short sintering time to save you up to 2.2 hours compared to leading pre-shaped cubic zirconia products. The manual shading and drying steps are also eliminated, saving up to a further 3.5 hours.

In addition, Lava Esthetic zirconia is easier to adjust and remove than conventional zirconia for a simpler professional workflow.

Start saving time without compromising on the quality of your restorations with Lava Esthetic zirconia from 3M Oral Care.

For more information, call 08456025094 or visit www.3M.co.uk/OralCare.

$3 \mathrm{M}$ and Lava are trademarks of the $3 \mathrm{M}$ Company.

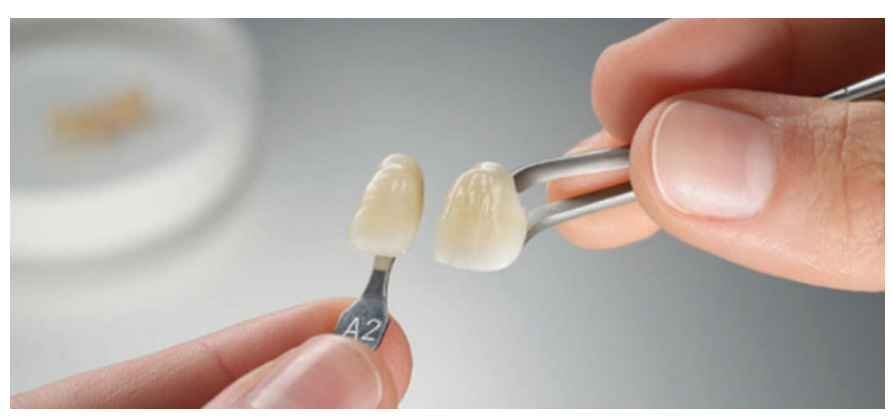

\section{A fully streamlined implant system}

BioHorizons is delighted to be bringing Camlog's iSy implant system to the UK and Ireland dental markets.

iSy, 'The Intelligent System' is a complete, fully streamlined implant system, with high primary stability and the capability to provide fully digitised restorations. This intelligent approach to workflow gives dentists superior time and cost efficiencies.

Everything needed for single implant surgery is contained within the all-in set, including single-patient form drill, iSy implant with pre-mounted base, two multifunctional caps, cover cap and gingiva former.

The implant's innovative design offers dentists improved performance:

- $2+2$ cutting groove design gives perfect tapping performance

- Fast osseointegration and stable bone apposition long-term thanks to the proven Promote Surface

- Pre-mounted implant base promotes transgingival healing

- One prosthetic platform - a standardised connection for all implants and prosthetics

- Click mechanism and matched components lead to time-savings.

For more information on the iSy implant system visit www.camlog.co.uk or contact the exclusive UK \& Ireland distributor BioHorizons: infouk@biohorizons.com, 01344 752560, www.biohorizons.com. 